\title{
137 \\ In and Out of the Box: Interaction Paradigms in Electronic Environments
}

\author{
Jacquelyn Martino and Lira Nikolovska \\ Philips Research, A division of Philips Electronics North America \\ 345 Scarborough Road \\ Briarcliff, NY 10510 USA \\ tel: 914.945 .6290 \\ e-mail: iam@philabs.research.philips.com \\ L.Nikolovska@DESIGN.CORP.philips.com
}

\begin{abstract}
This two-day cross-disciplinary workshop is for those who have developed alternative interaction paradigms in electronic environments. The goal is to exchange information on issues surrounding the design and theory of electronic environments. The design of these environments stimulates innovative interaction paradigms while considering the boundaries that the physical computer may or may not present. Interaction designers, architects, artists, industrial designers, computer scientists, writers, cinematographers, media theorists, cognitive and perceptual psychologists, ergonomists and educators all look at the problem of engaging the user and creating solutions for users of electronic environments from different points of view. In this workshop we discuss the concepts and ideas that lead these innovators to designing alternative interaction paradigms.
\end{abstract}

KEYWORDS interaction design, immersive virtual environments, information spaces, input devices, interaction models

\section{THEME OF THE WORKSHOP}

This two-day workshop focuses on cross-disciplinary discussion of topics relevant to alternative user interaction paradigms that occur both inside and outside of the boundaries defined by the physical computer or "the box." In contrast to traditional interaction paradigms (e.g. windows, menus and icons accessed by mice and keyboards), approaches that challenge the physical or metaphorical boundaries of the computer lead to alternative interaction paradigms. Workshop themes are:

1. Alternative interaction paradigms that may include 3D graphics, 2D graphics and/or original hardware configurations,
2. Approaches to designing and theorizing electronic environment creation and interactions within these environments.

In preparing for the workshop, participants should consider the following questions:

1. Does the computer impose boundaries? How are the activities that occur inside and outside the edges of the computer monitor mitigated by the interaction paradigm?

2. What are the approaches and methodologies for designing electronic environments and alternative interaction paradigms?

3. How can traditional approaches and methodologies from disciplines such as architecture, industrial design, graphic design, fine arts, cinematography, psychology 
and education be translated to the design of electronic environments and alternative interaction paradigms?

4. How is traditional training transformed by the ability to design electronic environments and alternative interaction paradigms?

5. What knowledge from real world experiences can be applied to the design of electronic environments making human interaction natural and intuitive?

Although the specific discussions will be shaped by the participants' positions, the organizing topics are as follows:

1. Innovative interaction paradigms in

- art, education, training and entertainment

- information visualization

- cyber architecture

- immersive and non-immersive virtual environments

2. Interfaces and interaction paradigms in and out of the box, including

- intelligent objects and intelligent spaces

- computer-augmented environments and devices

- innovative input devices

- immersive and non-immersive virtual environments

3. Applied design concepts and methodologies

4. Theoretical and conceptual design frameworks

5.Spatial perception and cognition, including reflections on issues of relationship between physical and abstract information configuration and comprehension

6. Visions for future research

\section{GOAL OF THE WORKSHOP}

Many solutions exist to the problem of designing and theorizing electronic environments. Solutions may be immersive or non-immersive, require conscious participation or be transparent to the user. The common thread, however, remains that computer users live in a spatial world with functional understanding of interaction in day to day life.
The goal of the workshop is to explore how diverse practitioners use this common understanding to manage electronic environment experiences through the development of their interaction paradigms. The issue at question is not if real world interaction is superior or inferior to electronic interaction, but to look critically into the potentials for designing and theorizing for the new electronic media that is already in use.

\section{Participants}

A maximum of sixteen workshop participants with demonstrable experience in developing and theorizing alternative interaction paradigms and who represent a wide variety of backgrounds will form three to four panels. The panels will be formed according to topics presented in position papers. Each panel will present to the remaining participants to foster interesting and provocative discussion.

\section{DESCRIPTION OF WORKSHOP ACtivities}

Day 1:

- General introduction position presentation by the organizers, with stress on the topics and goals of the workshop

- Position papers presented by individual workshop participants

Day 2:

- Topic-clustered panel sessions moderated by a predetermined participant. Position papers of the panelists will serve as a framework for discussion. Panel moderators are responsible for developing a set of questions based on panelists' topic expertise. The goal is to tackle the topic from individual perspectives while engaging other workshop participants in active discussion.

- Closing statement by organizers, shaped by the discussions that took place during the workshop. 